P. M. Whitman

1. Groups with a cyclic group as lattice homomorph, Ann. of Math. vol. 49 (1948) pp. 347-351.

G. ZAPPA

1. Determinazione dei gruppi finiti in omomorfismo strutturale con un gruppo ciclico, Rendiconti del Seminario Matematico della Università di Padova vol. 18 (1949) pp. 140-162.

2. Sulla condizione perchè un omomorfismo ordinario sia anche un omomorfismo strutturale, Giornale di Matematiche di Battaglini (4) vol. 78 (1949) pp. 182-192.

H. ZASSENHAUS

1. Lehrbuch der gruppentheorie, 1937.

UNIVERSITY OF ILLINOIS

\title{
SEMI-AUTOMORPHISMS OF SYMMETRIC AND ALTERNATING GROUPS
}

\section{FLORA DINKINES}

1. Introduction. In 1947, Irving Kaplansky [5 $]^{1}$ extended certain results of Ancochea [1] on semi-automorphisms of division algebras by redefining the concept of a ring semi-automorphism.

The definition which proved fruitful there is: A semi-automorphism of a ring is an additive automorphism $a \rightarrow a^{\prime}$ satisfying $(a b a)^{\prime}=a^{\prime} b^{\prime} a^{\prime}$. It has been established that every semi-automorphism of a division ring is an automorphism or an anti-automorphism, [5] and [4], and this result has found application in various places; for example, in [2]. In view of this application of ring semi-automorphisms it is not inconceivable that a similar type of mapping for groups may prove useful. Following the ring concept we define a semi-automorphism of a group to be a 1-1 mapping of the group onto itself such that $(a b a)^{\prime}$ $=a^{\prime} b^{\prime} a^{\prime}$ and investigate the question: Under what conditions is a group semi-automorphism an automorphism or an anti-automorphism?

This article is an excerpt from a thesis prepared under the direction of Professor Kaplansky which answers this question for the following classical cases: symmetric groups, alternating groups, dihedral groups, finitely generated abelian groups, and certain linear groups over a 2-dimensional vector space.

For brevity we shall say that a group $G$ is SA if every semi-automorphism of $G$ is an automorphism or an anti-automorphism. For

Received by the editors July 25,1950 .

${ }^{1}$ Numbers in brackets refer to the bibliography at the end of the paper. 
groups which are the direct product of noncommutative groups it is evident that there always exist semi-automorphisms which are automorphisms on one factor and anti-automorphisms on the other factor, hence the groups are not SA. However, the fact that indecomposability does not insure the SA property is evidenced by the dihedral groups which are not SA. One might conjecture that every simple group is SA. Our investigation shows no exception to this conjecture among the simple groups considered but it further shows that many groups which are not simple are SA. Aside from the complete results for symmetric and alternating groups given here we mention specifically only one further result; namely, the two-dimensional special linear group over an arbitrary field is SA.

2. Definitions and general lemma. Since Lemma 2 of [5] shows that if a semi-automorphism $S$ of a group sends the identity $e \rightarrow g$, then

(1) $g$ is in the center,

(2) $g^{2}=e$, and

(3) the mapping $S$ followed by $x \rightarrow x g$ is a semi-automorphism; the study of semi-automorphisms of groups can be reduced to those in which $e \rightarrow e$. We therefore formally state the definition to include this.

Definition 1. A semi-automorphism of a group $G$ is a 1-1 mapping of $G$ onto $G$ such that for the identity element $e$ and arbitrary elements $a$ and $b$ of $G$

$$
e^{\prime}=e
$$

and

$$
(a b a)^{\prime}=a^{\prime} b^{\prime} a^{\prime} .
$$

Definition 2. An element $x$ of a group $G$ is special if $x=a_{1} a_{2} \cdots a_{n}$ where $a_{i}$ is in $G$ and $\left(a_{1} a_{2} \cdots a_{n}\right)^{-1}=a_{n} \cdots a_{2} a_{1}$.

The following lemma is easily proved.

LEMMA 1. Under a semi-automorphism of a group $G$

(1) nth powers map into nth powers,

(2) inverses map into inverses, and

(3) if every element of $G$ is special, then conjugate classes map onto conjugate classes.

Proof of (3). Let $x$ and $y$ denote elements of $G$ and consider $\left(x^{-1} y x\right)^{\prime}$. Since $x=a_{1} a_{2} \cdots a_{n}$ where $\left(a_{1} a_{2} \cdots a_{n}\right)^{-1}=a_{n} \cdots a_{2} a_{1}$, we have 


$$
k=\left(a_{n} \cdots a_{1} y a_{1} \cdots a_{n}\right)^{\prime}=a_{n}^{\prime} \cdots a_{1}^{\prime} y^{\prime} a_{1}^{\prime} \cdots a_{n}^{\prime} .
$$

Then $a_{n}^{\prime} \cdots a_{1}^{\prime} a_{1}^{\prime} \cdots a_{n}^{\prime}=a_{n}^{\prime} \cdots\left(a_{1}^{2}\right)^{\prime} \cdots a_{n}^{\prime}=\left(a_{n} \cdots a_{1}^{2} \cdots\right.$ $\left.a_{n}\right)^{\prime}=e^{\prime}=e$. Therefore, $k=\left(a_{1}^{\prime} \cdots a_{n}^{\prime}\right)^{-1} y^{\prime} a_{1}^{\prime} \cdots a_{n}^{\prime}$ is a conjugate of $y^{\prime}$, and conjugate classes map onto conjugate classes.

We shall frequently use the fact that any element which can be expressed as the product of elements of order two is a special element.

Definition 3. A permutation is a 1-1 mapping of a set into itself.

Definition 4. For a given positive integer $n$, the finite symmetric group $S_{n}$ is the set of all permutations of $n$ objects.

Definition 5. For arbitrary infinite cardinals $\boldsymbol{N}$ and $\boldsymbol{N}^{\prime}$, the infinite symmetric group $S\left(\boldsymbol{N}, \boldsymbol{\aleph}^{\prime}\right)$ is the set of all permutations of $\boldsymbol{\aleph}$ objects which move less than $\boldsymbol{N}^{\prime}$ objects.

Definition 6. For any cardinal $\boldsymbol{\aleph}$, finite or infinite, the alternating group $A_{\aleph}$ is the set of all finite even permutations of $\boldsymbol{N}$ objects.

In the following proofs a symmetric group will be one satisfying Definitions 4 or 5 , and an alternating group will be one satisfying Definition 6.

3. The main theorem. Every semi-automorphism of a symmetric group or of an alternating group is an automorphism or an anti-automorphism.

LemMa 2. A semi-automorphism of a symmetric group maps conjugate classes onto conjugate classes.

Proof. Every cycle, finite or infinite, can be expressed as the product of elements of order two as follows:

$$
(\cdots 642135 \cdots)=[(12)(34) \cdots][(1)(23)(45) \cdots] .
$$

(This notation indicates the analogous product for finite cycles.) Since the cycles are disjoint, every element can be expressed as the product of two elements of order two. Part (3) of Lemma 1 then shows that conjugate classes map onto conjugate classes.

Lemмa 3. A semi-automorphism of an alternating group maps conjugate classes onto conjugate classes if the number of objects is greater than four.

Proof. This follows immediately from Lemma 1, since the 3-cycles in an alternating group generate the group and for $n \geqq 5$ any 3 -cycle $(i j k)=(i j)(t m) \cdot(i k)(t m)$.

LemмA 4. Under a semi-automorphism of $S_{n}$ or $A_{n}$ a 3-cycle can map into a product of 3-cycles only when $n=6$. 
Proof. The number of 3 -cycles is $n(n-1)(n-2) / 3$ and the number of products of $k 3$-cycles is $n(n-1)(n-2) \cdots(n-3 k+1) / 3^{k} k$ !. These are equal only when $k=2$ and $n=6$ [8]. Since 3-cycles are conjugate for all $n$ in $S_{n}$ and for $n \geqq 5$ in $A_{n}$ and conjugate classes map onto conjugate classes, the lemma is proved.

Lemma 5. Let $S$ and $A$ denote symmetric and alternating groups, respectively, which contain an infinite number of elements. Then under a semi-automorphism of $S$ or of $A$ a single 3-cycle must map into a single 3-cycle.

Proof. Let $B$ denote the set of single 3-cycles in $S$ or in $A$. Then the only possible types of products of the form $a b a$ with $a, b \in S$ or $A$ are as follows:

$$
\begin{aligned}
& (123)(123)(123)=(1), \\
& (132)(123)(132)=(132), \\
& (124)(123)(124)=(132), \\
& (142)(123)(142)=(12)(34), \\
& (145)(123)(145)=(15234), \\
& (456)(123)(456)=(123)(465) .
\end{aligned}
$$

We now let $k$ be a fixed integer $\geqq 2$ or let $k$ be a fixed infinite number, and let $A_{k}$ denote the set of all products of $k$ disjoint 3-cycles. If a single 3-cycle maps into a product of $k 3$-cycles, then $B$ must map onto $A_{k}$, since $A_{k}$ forms a single conjugate class. The lemma will be proved if there is an $a b a$ product with $a, b \in A_{k}$ having an order different from all orders in (1).

We use the same notation for $k$ finite or infinite and let

$$
\begin{aligned}
& a=(123)(546)(x y z)(r s t)(u v w) \cdots, \\
& b=(132)(567)(x y z)(r s t)(u v w) \cdots .
\end{aligned}
$$

Then $a b a=(123)(47)(56)$ is of order six and the lemma is proved.

LEMMA 6. The elements of order three and the elements of order two of a symmetric group generate the group under the operation aba.

Proof. We shall show first that any finite cycle $c$ of degree greater than 3 can be expressed as $a b a$ where $a$ is a product of disjoint 3-cycles and $b$ is a product of disjoint transpositions and the only numbers that occur in $a$ and $b$ are those in $c$.

We now show how to produce an $n$-cycle for each $n$.

(1) Let $n=3 k$. For $n \geqq 2$ the $a b a$ product for 


$$
\begin{aligned}
a & =(123)(456)(789) \cdots(3 k-2,3 k-1,3 k), \\
b & =(34)(67) \cdots(3 k-3)(3 k-2)
\end{aligned}
$$

is given by the formula

$$
(13258,11, \cdots, 3 k-1,3 k-2,3 k, \cdots, 10,12,7,9,4,6) ;
$$

that is, the first four numbers are 1325 , these are followed by the middle number of each cycle in regular order, and these are followed by the first and third numbers in each cycle where the cycles are considered in reverse order and the first cycle is omitted.

This formula holds for $k=2$ since

$$
(123)(456)(34)(123)(456)=(132546) \text {. }
$$

We assume then that it holds for some $k$ and consider the $a b a$ product for

$a=(123)(456) \cdots(3 k-2,3 k-1,3 k)(3 k+1,3 k+2,3 k+3)$,

$b=(34)(67) \cdots(3 k-3,3 k-2)(3 k, 3 k+1)$.

This gives $(13258, \cdots, 3 k-1,3 k+2,3 k+1,3 k+3,3 k-2,3 k, \cdots$, $10,12,7946)$; hence the formula holds for $k+1$. Similar proofs can be given for $n=3 k+1$ and $n=3 k+2$.

We next show that an infinite cycle $D$ can be obtained as an $a b a$ product of transpositions and 3-cycles which contain only the numbers of $D$.

$$
D=(\cdots, 12,10,8,6,4,2,1,3,5,7,9,11, \cdots)=a b a,
$$

where

$a=(153)(472)(896)(12,11,10)(16,13,14)(20,15,18)(24,17,22) \cdots$,

$b=(34)(28)(6,12)(10,16)(14,20)(18,24) \cdots$.

Now let $E$ be any permutation not consisting entirely of 3-cycles and containing at least one $n$-cycle for some $n \geqq 3$. Since its cycles are disjoint, there will be no overlapping of numbers if $a$ and $b$ are chosen as follows:

Let $a$ consist of (i) the inverses of all 3-cycles appearing in $E$ and (ii) all 3-cycles needed to express all other cycles as described in the first part of this proof.

Let $b$ consist of (i) all transpositions appearing in $E$ and (ii) all transpositions needed to express all other cycles as in the first part of this proof. Then $E=a b a$. 
Lемма 7. The elements of order three and the elements of order two of an alternating group generate the group under the operation aba.

Proof. We need to observe merely that the formulas for finite cycles $c$ of Lemma 6 involve even products of transpositions when $c$ is an even $n$-cycle and odd products of transpositions when $c$ is an odd $n$-cycle. Hence the element of order two needed for any even permutation is an even permutation.

Proof of the MaIN Theorem. We shall first consider all semiautomorphisms which send a single 3-cycle into a single 3-cycle. This will include all semi-automorphisms except those of $S_{6}$ and $A_{6}$ which send a 3-cycle into the product of two 3-cycles. The theorem will be proved for this case if the mapping can be reduced to the identity mapping by applying normalizations which are automorphisms or anti-automorphisms. We proceed to this end.

If $(123)^{\prime}=(i j k)$ under a semi-automorphism $T(X)=X^{\prime}$, we normalize to the identity by applying the inner automorphism which sends $(i j k)$ into (123). Since inverses map into inverses, $(132)^{\prime}=(132)$.

Then (124) must have exactly two numbers in common with $(123)^{\prime}=(123)$ and these numbers must occur in the same order as in $(123)$, for if $(124)^{\prime}=(1 t 2),(1 \mathrm{tm})$, or $(t m n)$, a contradiction arises as follows:

$$
\begin{aligned}
{[(123)(124)(123)]^{\prime}=(142)^{\prime} } & =(123)(1 t 2)(123)=(12)(3 t), \\
& \text { or }=(123)(1 \mathrm{tm})(123)=(13 \mathrm{tm} 2), \\
& \text { or }=(123)(t m n)(123)=(132)(t m n) .
\end{aligned}
$$

Therefore, $(124)^{\prime}=(12 t),(23 r)$, or $(1 s 3)$.

In each case there exists an automorphism of the form $x \rightarrow y^{-1} x y$ (where $y$ may be in the corresponding symmetric group if the alternating group is being considered) which leaves (123) fixed and sends $(124)^{\prime}$ into (124). This normalizes the mapping to the identity on (123) and (124).

Since $(125)^{\prime}$ must have exactly two numbers in common with each of the elements (123) and (124) and these must be arranged in the same order as in (123) and in (124) we know that

$$
(125)^{\prime}=(12 t) \text {. }
$$

If $t \neq 5$ the inner automorphism $x \rightarrow(5 t) x(5 t)$ normalizes the mapping to the identity on (123), (124), and (125). Similarly, we can obtain $(126)^{\prime}=(126)$.

Let $B$ denote the set of elements of the form (12r). The preceding 
argument shows that every element in $B$ has an image in $B$. We now show that if an element $z$ is not in $B$, it cannot have an image in $B$.

We consider the following cases:

(a) $z$ contains both 1 and 2 and is therefore of the form (1j2).

(b) $z$ contains only one of these numbers and is of the form $(1 j k)$.

(c) $z$ contains neither of these numbers and is of the form (ijk).

In all cases if $z^{\prime}=(12 t),\left(z^{-1}\right)^{\prime}=(1 t 2)$, and a contradiction arises as follows:

Let $(12 s)$ be such that $s=3,4,5$, or 6 and $s \neq i, j$, nor $k$. Then

$$
\begin{aligned}
{[(12 s) z(12 s)]^{\prime} } & =[(12 s)(1 j 2)(12 s)]^{\prime}=[(12)(j s)]^{\prime} \\
& =(12 s)(12 t)(12 s)=(1 t 2), \\
\text { or } & =[(12 s)(1 j k)(12 s)]^{\prime}=(1 s j k 2)^{\prime}=(1 t 2), \\
\text { or } & =[(12 s)(i j k)(12 s)]^{\prime}=[(1 s 2)(i j k)]^{\prime}=(1 t 2) .
\end{aligned}
$$

This shows that the mapping on $B$ is $1-1$ onto. $\left(12 r_{1}\right) \rightarrow\left(12 s_{1}\right)$, $\left(12 r_{2}\right) \rightarrow\left(12 s_{2}\right), \cdots,\left(12 r_{\alpha}\right) \rightarrow\left(12 s_{\alpha}\right), \cdots$.

Let $h$ be the permutation $r_{\alpha} \rightarrow s_{\alpha}$. Then $x \rightarrow h^{-1} x h$ normalizes the mapping to the identity on $B$ and on the inverses of $B$.

We now consider (143)'. Since (143) has two letters in common with each of the elements (142) and (123), it must contain two letters from each in the same order as they occur in (142) and in (123). This is possible if and only if $(143)^{\prime}=(143)$ or (234).

If $(143)^{\prime}=(234)$, the anti-automorphism $x \rightarrow(12) x^{-1}(12)$ normalizes the mapping to the identity on $B$ and on the element (143).

In order to show that all 3-cycles map identically it suffices to show that each 3 -cycle or its inverse can be produced from the elements in $B$ and the element (134) under the $a b a$ operation.

Since $(12 r)(1 s 2)(12 r)=(12)(r s)$ and $(12)(34)(134)(12)(34)=(243)$, this is true for $n=4$.

If $n \geqq 5,(12)(4 i)(134)(12)(4 i)=(23 i)$ for $i \neq 1,4$, but we have (231) and (234); therefore, we have this product for all $i$.

Then $(12)(3 j)(23 i)(12)(3 j)=(1 j i)$ for $i, j \neq 2,3$. In order to obtain (1ij) for $i=3$ we use

$$
(12)(4 j)(234)(12)(4 j)=(13 j) \text {. }
$$

Then $(1 j i)(1 i 2)(1 j i)=(1 i)(2 j)$ and

$$
(1 i)(2 j)(12 k)(1 i)(2 j)=(i j k), \quad i, j \neq 1,2 .
$$

Since we have all $(1 j i)$, we need only produce all $(2 j k)$. This we obtain from

$$
(12)(i k)(1 j i)(12)(i k)=(2 j k) \text {. }
$$


Therefore, all single 3-cycles map identically. We now show that all elements map identically.

Let $x$ be a product of disjoint transpositions and let $y=x^{\prime} \neq x$. Then some transposition $(i j)$ is contained in $x$ or in $y$ but not in both. Let $z=(i j k)$, then $(x z x)^{\prime}=x^{\prime} z^{\prime} x^{\prime}=y z y$. Since $x$ and $y$ are of order two, $x z x$ and $y z y$ are conjugates of $z$. One of these products will be of the form (jir) and the other will be different, since it will fail to contain both $i$ and $j$; or, if it contains both, will send $i$ into $j$. Therefore $x^{\prime}=x$ for all transpositions.

Let $x$ be a product of a disjoint 3-cycles, then

$$
x=(123)(456)(789) \cdots(i j k) \cdots,
$$

and $x^{\prime}=y$ is also a product of 3 -cycles. Let $z=(12)(45)(78) \cdots$ (ij) $\cdots$. Then $z x z=z$ and since $(x z x)^{\prime}=x^{\prime} z^{\prime} x^{\prime}=y z y, y$ must be of such form that $y z y=z$. This will happen if and only if for every transposition $(i j)$ in $z$ one of the following is true:

(a) $i$ and $j$ do not appear in $y$.

(b) $y$ contains a 3 -cycle containing both $i$ and $j$.

If now we repeat the argument with $z=(13)(46)(79) \cdots(i k): \cdots$, we conclude that if $y$ contains a number in a 3-cycle ( $i j k)$ of $x$ it contains $(i j k)$ or $(j i k)$.

If $y$ does not contain the letters of a given 3-cycle, say the cycle (123), then a contradiction arises as follows:

Let $z=(45)(78) \cdots(i j) \cdots$. Then $x z x$ is of order 6 while $y z y=z$. Therefore, for a given 3 -cycle in $x, y$ contains this 3-cycle of its inverse, and conversely.

It suffices to consider the cases

$$
\begin{aligned}
& x=(123)(456) \cdots, \\
& y=(132)(465) \cdots, \\
& x=(123)(456) \cdots, \\
& y=(132)(456) \cdots,
\end{aligned}
$$

For each case let $z=(234)$, then $x z x$ is of order 15 or 5 and $y z y$ is of order 12 or 4 . This is a contradiction. Therefore $x^{\prime}=x$.

Since for the symmetric and the alternating groups the elements of orders two and three generate the group under $a b a$, the mapping is the identity on all elements, and the theorem is proved for the case where a single 3-cycle maps into a single 3-cycle.

Finally, let $V$ be a semi-automorphism of $S_{6}$ (or of $A_{6}$ ) which sends a 3-cycle into a product of two 3-cycles. There exists [3] a known 
automorphism $W$ of $S_{6}$ (or of $A_{6}$ ) which sends a product of two 3cycles into a single 3-cycle.

Then $U=W V$ is a semi-automorphism which sends a 3-cycle into a 3-cycle, hence, by the first part of this proof, is an automorphism or an anti-automorphism. Therefore $V=W^{-1} U$ is an automorphism or an anti-automorphism.

\section{BIBLIOGRAPHY}

1. G. Ancochea, On semi-automorphisms of division algebras, Ann. of Math. vol. 48 (1947) pp. 147-153.

2. Jean Dieudonne, On the automorphisms of the classical groups, Memoirs of the American Mathematical Society, no. 2, 1951.

3. O. Hölder, Bildung zusammengesetzter Gruppen, Math. Ann. vol. 46 (1895) pp. $321-422$.

4. Loo-Keng Hua, On the automorphisms of a sfield, Proc. Nat. Acad. Sci. U.S.A. vol. 35 (1949) pp. 386-389.

5. Irving Kaplansky, Semi-automorphisms of rings, Duke Math. J. vol. 14 (1947) pp. 521-525.

6. J. Schreier and S. Ulam, Über die Automorphismen der Permutationsgruppe der natïrlichen Zahlenfolge, Fund. Math. vol. 28 (1937) pp. 258-260.

7. - Über die Permutationsgruppe der natïrlichen Zahlenfolge, Studia Mathematica vol. 4 (1933) pp. 134-141.

8. I. E. Segal; The automorphisms of the symmetric group, Bull. Amer. Math. Soc. vol. 46 (1940) p. 565.

UNiversity of Chicago 